to heal, became very fetid, and he went down under the devouring fire of septic poison. In the other four cases antiseptics were rigidly adhered to, the wounds were free from odor, healthy in appearance, and the process of repair went steadily on to recov ery. In Case $x$, could the exact condition of the injured bones in the left leg have been known, a synchronous amputation might have saved the patient's life. In treating these severe injuries the following are some of the precautions necessary:

I. Close attention to all the details. Keep all general attendants away from the wounds. 2. Absolute rest at the point of injury. 3. Look closely after the general health of the patient. Don't take anything for granted. 4. See that a proper mental and physical balance is maintained.

Absolute rest at the point of injury cannot be too strongly emphasized, since success or failure in treatment depends largely on the attention that is given to this principle.

In this connection I desire to call attention to a device which has been of inestimable value to me in the treatment of these cases. This apparatus is original with Dr. W. J. Herdman, of Ann Arbor, Mich., and is known as "Herdman's Lifting Device." This apparatus is simple in construction, durable and cheap. It is made of ordinary gas-pipe and wrought iron, and any ordinary machinist can make one. One man can lift a patient from the bed without causing any pain whatever, and at the same time greatly add to the comfort of an injured person, while a change of bedding or other clothing is being made. The following cut will fully illustrate the construc tion of the device. One thing to be remembered in constructing this device is, that the cross-bands must be the same in length as the distance between the cradle-hooks.

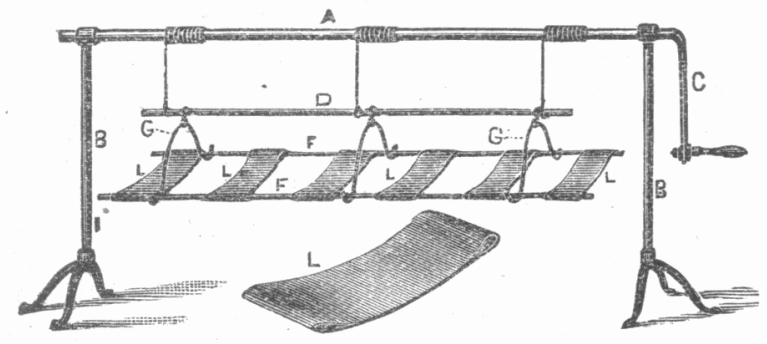

HERDMAN'S LIFTING DEVICE.

A, cross bar: B, legs; C, crank; D, cradle; L, L, L, L, cross bands, loop stitched at ends for sliding bar; F, sliding bar; G, cradle hooks. The device should be long enough to swing over the bed. The cradle is then lowered, the bands passed under the patient, the sliding bars passed through the looped ends of bands, then into the hoo
turns at the crank lifts the patient bodily from the bed.

Finally, in the language of a well-known writer :2 "In the light of the researches of experimenters in the field of bacteriological science, and the experience of those who, acting upon the suggestive results derived from these experiments, treat wounds antiseptically, in my opinion, the surgeon who does not take into account every possible source of danger that may overtake his patient, including in this the

8 Geo. R. Fowler, M.D., in New York Medical Journal. noxious influences arising from the entrance of germs into the tissues, is criminally negligent, and directly responsible, both to his own conscience and to the world at large."

I am greatly indebted to Dr. H. N. Spencer for valuable services rendered during the treatment of these cases.

\section{ON THE TREATMENT OF FELON WITHOUT INCISION.}

Read in the Section on Medicine, Materia Medica and Therapeutics, at the Thirty.Eighth Annual Meeting of the American Medical Association, Fitne 7, 1887.

BY L. DUNCAN BULKLEY, A.M., M.D., ATTENDING PHYSICIAN TO THE NEW YORK SKIN AND CANCER HOSPITAL, ETC.

The teachings of instructors in surgery, and also those in the text-books on the subject, are all to the effect that felon, paronychia, or whitlow, will require sooner or later a free, deep incision, even to the bone, and that in the majority of cases the sooner this is done after the diagnosis is made, the better. I believe, also, that careful inquiry would show that this is the almost universal practice both of those who follow surgery more particularly, and of general practitioners. But little is found in writings in regard to the medical and internal treatment of these cases, other than the suggestion that tonics are often needed; and the almost universal recommendation is that poultices should be applied, both before and after the incision.

Having had quite a considerable number of cases of felon under my care in public and private practice, some of them being very severe, and having pursued a very different course of treatment from that usually followed, as far as I can learn, I beg to present the matter briefly, in order that others may make trial of the same methods, which, as I believe, have advantages worthy of consideration.

I will not attempt to enter into any anatomical or pathological study of the parts concerned, or of the affection under consideration, but in order not to be misunderstood I wish to state that by felon and whitlow I refer to the cases familiar to all, in which there is deep-seated inflammation, mainly of the ends of the fingers, tending to penetrate even to the bone, and ultimately, if unchecked, to cause necrosis of the same. Some of my cases have been light and have remained superficial; others have been first seen when the inflammation had already penetrated deeply, when the finger was hot, tense and hard, and exceedingly painful on pressure, and even after the patient had already passed sleepless nights with the throbbing pain. But for many years I have never incised a case, other than a possible slight prick, without pain, into dead skin after the pus had become superficial; nor have I ordered a poultice for such a case within the same period of time.

While a certain number of cases of whitlow seem to have their origin deep in the tissues of the finger, or even in the periosteum, in the majority of cases the inflammation appears to originate, and probably does, in a relatively superficial injury, as a prick of a pin, or needle, or nail, a scratch, or other appar- 
ently trivial accident, to which little or no attention is paid at the time. As is well known, when the inflammation has once started it proceeds with considerable rapidity, and three or four days after its beginning the patient may be suffering agony from the deep-seated, tense, throbbing pain, and the entire tissues of the finger are found to form a firm, hard mass, exquisitely sensitive to pressure.

The reason for the ready and rapid extension of the inflammation from the superficial to the deep structures is undoubtedly found in the anatomical peculiarities of this part, which consist in the existence of fibrous threads extending from the skin to the periosteum, binding all the parts firmly together; along these connective tissue bands the inflammation travels from without inward with ease, and it is also because of the existence of these connecting fibres that, under the treatment now proposed, in a deepseated inflammation, originating even in the sheath of a tendon or the periosteum, the pus may find exit without causing great damage, as will be presently shown.

As an element in the production of whitlow the general condition of the patient must always be taken into consideration, and especially that unknown but still recognized state manifested by the tendency to form pus, the pyogenic habit, as it was formerly called. Every prick of a needle or nail in the end of the finger does not result in a felon; nor in a given number of subjects could it be produced in any great proportion of instances by any amount of injury to the finger tip; nor can it be produced at will even in those who are ever so much run down in health, as in so many other conditions of disease, some predisposing cause which causes what might otherwise be an innocuous prick to become the beginning of a serious inflammatory process. Its localized phenomena would almost point to an infec. tious origin, received with the wound, were it not that many cases originate deeply without external puncture.

The treatment which I would advocate, and which has proved valuable in my hands, consists both of general and local measures, and has for its aim the checking of the inflammatory and suppurative process rather than the encouragement of suppuration, as is ordinarily done by poultices.

Patients even with a beginning felon are commonly found to be in a state of lowered vitality, often with more or less of a sluggish condition of the digestive organs, coated tongue, loss of appetite, etc. Unless it is contraindicated I generally begin the treatment with a mild cathartic, the following being that commonly employed:

R. Ext. colocynth comp.

Mass hydrarg................... àa gr, x

Pulv. ipecacuan .................... gr, ii

m. Div. in pil. No. iv. Sig: Take two at night and two on the second night after.

A tonic is administered from the first, one containing iron being preferred; that usually given is a combination known as Startin's mixture, which has considerable power in controlling inflammatory affections of the skin. The formula of this is as fol- lows, the proportions being somewhat altered to suit individual cases :
R. Magnesii sulphatis...................

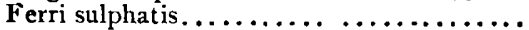

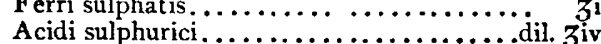

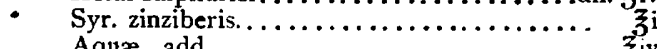

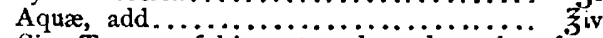

m. Sig: Teaspoonful in water, through a tube, after eating.

In addition to this it is my custom to administer the sulphide of calcium (calx sulphurata) from the beginning to the end of the treatment. I usually give it in the form of $1 / 4$. grain gelatin coated pills, one being given every two hours irrespective of food or other medicine. In order to have any good effect from this latter drug it is essential that it should be fresh and pure, for, as is well known when exposed to the air it rapidly loses its free sulphur and becomes converted into the inert sulphate of zinc, or gypsum. It cannot, therefore, be well given in powders or tablets, nor even in extemporaneous pills, but is best administered in those well coated with gelatin, which preserves the drug unaltered; it is well, however, to test the pills by biting them, when the characteristic odor of sulphuretted hydrogen becomes at once noticeable if the article is good.

Some attention to the diet should also be paid in these cases, and specific directions should be given. Alcohol in all forms should be absolutely interdicted, and the malted liquors appear to be also very harmful. The diet should be full and nourishing, but not stimulating. Milk is often given, sometimes in the form of punch and egg-nog, but, as I have elsewhere explained very fully, I believe that milk is not a desirable article to take in connection with other food, and my constant direction is that it should be avoided at the meals. On the other hand, it serves a most admirable purpose in raising the vital tone of these patients if given separately and alone, in the interval between the meals, preferably one hour before each meal, and late at bed time, avoiding, however, taking it within half an hour of the granules, or of any other substance in the stomach. Tea and coffee may be taken in moderation, but unnecessary and indigestible articles should be avoided.

The local treatment of felon which I have followed has the merit of great simplicity and is one which can be readily followed out by every one. It consists simply in the constant any very thorough envelopment of the affected part in a protective ointment from the beginning to the end of treatment. The application used is the diachylon ointment of Hebra, which, when properly prepared, forms a most agreeable and soothing dressing.

Of late years it has been suggested to make the diachylon ointment by melting lead plaster with various oils, or vaseline, and much that is supplied on prescription will be found to be prepared in this manner. But this by no means answers as well in many conditions of the skin as that prepared according to the original formula given by Hebra, probably because the added portion of the oil has not undergone decomposition with the litharge. I am therefore very particular to have the ointment prepared according to the original formula, which is as follows : 
R. Olei olivarum optimi.............

Plumbi oxidi.................... $\xi_{\text {iij }} \xi \mathrm{vj}$

Olei lavandulæ.................. 3 ij

m. Add the oil to two pounds of water and heat it with constant stirring; the litharge is to be slowly sifted in while it is well sturred, fresh water being added as required. The ointment is to be stirred until cold, and the oil of lavender then added. In winter a slightly larger quantity of oil is required to make a soft ointment.

When properly made according to the above directions the ointment is soft and unctious, and not very sticky. It is to be spread upon the woolly side of lint very thackly, even a quarter of an inch or more in thickness, so as to completely envelop the end of the finger in a thick mass of the ointment; this is then lightly bound on and left undisturbed as long as possible; generally it is best to renew the application twice daily, and if there is no discharge the same dressing may have fresh ointment spread upon it, and be replaced quickly. The affected part should not be handled at all, nor the ointment scraped off even, the idea being simply to keep it continuously in the mass of bland, protective and absorptive ointment, to allow the inflammatory process to subside.

The first application of the ointment is described as being soothing and pleasant in the extreme; in mild cases just beginning it allays the irritation and pain at once, and even in those who had had sleepless nights from the pain, I have had them tell me that the relief was almost complete, and that they had slept the night after the application although in one or two instances the relief was more gradual.

Under the treatment above described some suppuration commonly takes place. In lighter cases, where the inflammation has begun superficially, one or more small points appear beneath the epidermis, which either break themselves or may be opened absolutely without pain through a slight prick in the dead covering. In those where the disease has begun more deeply, or where it has penetrated to some depth before treatment, the progress is more slow, and $a$ week may be taken before the pus comes near the surface; but then it shows itself also in one or more points, never very large, and the discharge may be hastened by a slight prick through the dead epidermis.

In one or more cases under my care small spiculæ of bone have been discharged, but still the case progressed satisfactorily without deep incision. After the opening the ointment is to be still continued in the same manner, the dressings being renewed more often if there is much discharge, until finally the openings close and the parts resume a normal condition.

The duration of the treatment of these cases compares very favorably with that of those treated in the ordinary manner with poultices and incision. I do not think that any of them have been obliged to use the ointment more than three weeks, and in the milder cases all trouble has sometimes subsided in a week or ten days.

My first cases were treated in this manner fully ten years ago, since which time I think I have applied the treatment to a dozen or more cases in pub- lic or private practice. I cannot, of course, vouch for all the cases I have seen, as to the final results, as all know the uncertainty attending out-patients in hospital practice; but I know of the complete success in a number of this class, and could give histories from notes of cases seen in private practice if time permitted.

A single case may be briefly mentioned: Mr. J. B., a gentleman aged 38 , is now under treatment for another. trouble, two years after his felon, for which I treated him in this manner. In this case there had been intense, deep pain, which was relieved almost entirely by the ointment. About a week after beginning treatment pus was discharged and several spiculæ of bone followed, but all healed quite rapidly, and now, when examined recently, the scar on the right forefinger is seen to be supple, and the integrity of the finger perfectly preserved.

I am quite aware that there may be cases in which this plan of treatment may not be applicable, but such have not as yet come under my observation. I shall look with interest for the experience of those who have opportunities of seeing more cases, and more severe ones, perhaps, than have come under my care, and feel confident that if the plan of treat ment $I$ have sketched is carried out thoroughly, it will be found to give good results certainly in a share of cases, and will certainly prove more acceptable to the patient than the annoying application of poul. tices and the much dreaded incision with the knife.

\section{DISCUSSION.}

Dr. Norman Bridge, of Chicago, had used vaseline, cosmoline and belladonna applications.

DR. NORTH, of Waterbury, had immersed the finger in a strong solution of iodine, where pus was not already formed, with good results. He immersed the finger three times a day for five minutes each time.

DR. Powell, of Indiana, had used oleate of morphia to relieve pain, keeping some constantly applied. It has given great relief.

Dr. ZeIsLer, of Chicago, said that ichthyol relieves pain to a great extent, even if it does not prevent suppuration.

Dr. Loss, of Pennsylvania, has dipped the finger in hot water for a half hour and then dipped it in collodion.

DR. C. C. P. Silva, of Chicago, said that water at a high temperature will prevent suppuration if abscess is in its incipiency and superficial. Water will not do so if the process is deep-seated.

Dr. Porter, of New Hampshire, had used water and iodine. For last five years he has used iodine and then pressure, by a plaster and roller bandage, using as much pressure as the patient will bear without severe pain. Pressure on the muscles may prevent congestion and suppuration. 